\title{
Numerical and Experimental Investigation of Fishnet-based Metamaterial in a \\ X-Band Waveguide
}

C .Sabah* and H. G. Roskos

Physikalisches Institut, Johann Wolfgang Goethe-Universität, Max-von-Laue-Str. 1, D-60438 Frankfurt am Main, Germany

* Corresponding Author: Sabah@Physik.uni-frankfurt.de

\begin{abstract}
In this paper, a fishnet-based metamaterial for the microwave X-Band frequencies is introduced and investigated both numerically and experimentally. The main innovation introduced here is that the structure is composed of dielectric and metallic parts in such a way that there is no connection between the metallic part of the metamaterial and the waveguide. The homogenous effective medium theory is employed for the characterization of the structure. A left-handed region is identified using the simulated and experimental results. The performance of the structure is described by a figure-of-merit calculation. The measured results are in good agreement with the simulated ones which show that the proposed metamaterial operates well like a fishnet metamaterial at around $10 \mathrm{GHz}$.
\end{abstract}

Keywords: Metamaterial, fishnet, negative refractive index, single and double negative, left-handed, microwave, waveguide experiment.

PACS: $41.20 . J b$, 81.05.Xj, 78.67.Pt 


\section{Introduction}

Artificial metamaterials (MTMs) are composite structures that have been engineered to have unusual electromagnetic properties, such as simultaneous negative permittivity and permeability, which are not readily available in nature. They have attracted considerable interest due to their potential applications in several fields of science and engineering $[1,2]$. Such MTMs can be constructed using a dielectric host and metal inclusions. They have been intensively studied in the form of various periodical combinations $[1,2]$. Among them, one of the most promising structures is the so-called fishnet MTM which consists of stacked dielectric and metallic layers with two-dimensional periodic holes in the metal layers [3-15]. Because of its superior performance, many researchers have focused on the investigation of the fishnet MTM to characterize and synthesize new combinations. The fishnet structure can be treated as an array of thin wires which are continuous along the electric field direction and short slab and/or cut wire pairs as magnetic resonators which are contiguous along the magnetic field direction. Under certain conditions, the structure can provide a negative refractive index at around the transmission peak.

In the present study, the electromagnetic response of a fishnet-based MTM is investigated, both numerically and experimentally, for X-band frequencies ( $8 \mathrm{GHz}$ to $12 \mathrm{GHz}$ ). The structure is designed for the X-band rectangular waveguide (WR-90) and simulated using a full-wave electromagnetic solver based on the method of moments. The MTM layers are manufactured using low-cost printed circuit board production and the experiment is performed using a network analyzer and a WR-90 waveguide by placing the stacked layers into the waveguide. In the literature, the published fishnet MTMs are formed from continuously metallized mesh-like structures which are positioned to be perpendicular to the propagation direction of the wave. The main goal of this study is to explore the physical characteristics of the fishnet-based MTM formed by non-continuous metallization (see Figure 1: the metallic part is not touching the walls of the waveguide), and to tune the negative response of the permittivity and permeability onto each other.

Note that stacked fishnet MTMs composed of a periodic array of perforated conductor screens with continuous metallization have been studied numerically in Refs. 11 and 
15 based on waveguide theory. Experimental studies with waveguides loaded with multilayer fishnet MTMs have not been performed because of the difficulties arising for the interpretation of the data as a result of the continuous metallization. However, in our case, the proposed structure with non-continuous metallization was studied both numerically and experimentally. The key benefit of the new design with noncontinuous metallization is that it allows to take advantage of the method of images for the retrieval of the effective parameters. In this case, the effective medium theory can be applied easily and the proposed waveguide system loaded by a small metamaterial sample is adequate to carry out the extraction procedure. Both in the simulation and experiment, the reflection and transmission characteristics of the designed structure are obtained and then the effective material parameters are retrieved based on the Nicolson-Ross-Weir (NRW) procedure $[16,17]$. The stacked structure is assumed to be effectively a homogeneous medium because the wavelength of the propagating wave through the fishnet-based MTM is much larger than the periodicity of the structure in the propagation direction.

Note that, to our knowledge, the present experiment is the first experimental verification of simulations for fishnet-based MTMs in a waveguide. The data show that we succeed to achieve simultaneously negative permittivity and permeability in a certain frequency band. It is observed that the simulation and experimental data agree with each other quite well.

\section{Numerical Simulation and Experiment}

The schematic representations of the experiment and the front view of a single layer of the MTM structure are shown in Figure 1. The FR4 substrate has a thickness of $\lambda / 60$ ( $\lambda$ is the vacuum wavelength at the operation frequency) and precisely fits WR90. The metallic part, which is centered on FR4 and not connected to the waveguide walls, is made of copper with a conductivity of $5.8 \times 10^{7} \mathrm{~S} / \mathrm{m}$ and a thickness of $18 \mu \mathrm{m}$ which is larger than the skin depth at $10 \mathrm{GHz}$. The metallic part has the following dimensions: $w=2 \lambda / 3, l=\lambda / 3, d_{1}=\lambda / 40$, and $d_{1}=2 d_{1}$. The periodicity along the propagation direction is equal to the total thickness of the single MTM layer (substrate and copper) which is much smaller than the operation wavelength. The substrate is chosen to be as thin as possible to minimize the dielectric loss. The aim of the 
optimization which led to the selected dimensions was to have a double negative window at around $10 \mathrm{GHz}$.
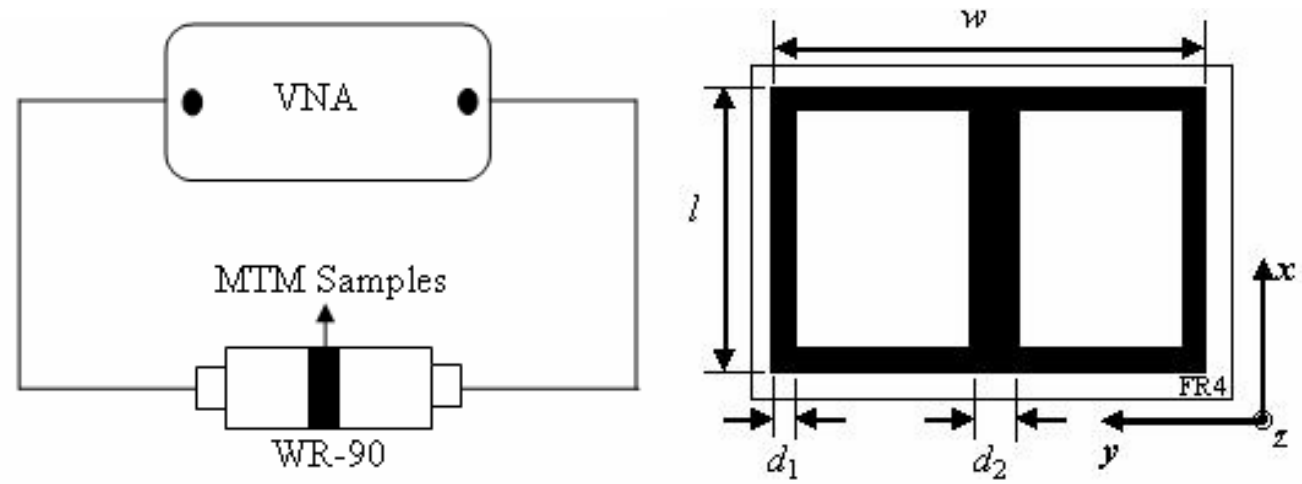

Figure 1: Schematic representations of the experimental set-up (left side) and a single layer of the fishnet-based MTM (right side). VNA stands for vector network analyzer. In the sketch of the MTM, the white part represents FR4 and the black part copper.

The structure was simulated using EEsof EDA design and simulation software. Waveguide boundary conditions were used for the side walls, and open boundaries for the waveguide ports for the excitation and detection of electromagnetic waves. Both in the simulation and the experiment, a 15-layer MTM structure was used and arranged as copper-FR4-copper-FR4-copper-... The electromagnetic wave propagates in the z-direction (normal to the plane of the fishnet-based MTM), and the electric and magnetic fields are oriented along the $\mathrm{x}$ - and y-directions, respectively. In this case, one obtains a magnetic response by the substrate-separated double-strips oriented in y-direction. The electric response is dominated by the strips in $\mathrm{x}$-direction (acting as a dilute Drude metal). Double negative and fishnet behavior can be achieved if a sufficient number of layers are stacked onto each other [3-15]. Usually, the magnetic response can be attributed to the excitation of a virtual current loop between the strips perpendicular to the magnetic field. These currents should be strong enough to be able to neutralize the magnetic field and produce a resonance displaying negative effective permeability. Simulations show that in our structure, the currents on opposite metallic surfaces are anti-parallel to each other at the resonance frequency, and hence the currents in each virtual metallic loop are circulating which is shown in Figure 2. This confirms the explanation given above and gives a clear identification of the character of the resonance. As a consequence, a negative 
permeability arises at the resonance. Note that each metal layer contributes to current loops with regard two adjacent dielectric layers. The two current contributions on each layer are opposite to each other, hence the total current is reduced which is not shown here. Therefore, the magnetic response of the whole stack is a collective response of all layers. At a stop-band frequency, it is observed that the currents on the metallic parts are in-phase and symmetric (data not shown). Consequently, the structure behaves like an ordinary structure comprised of a combination of dielectrics and conductor elements, and no artificial magnetism is observed. Note that the effective permittivity is achieved by the interaction of the field with the metallic parts which means it is controlled by the dimensions of the strips and periodicity.
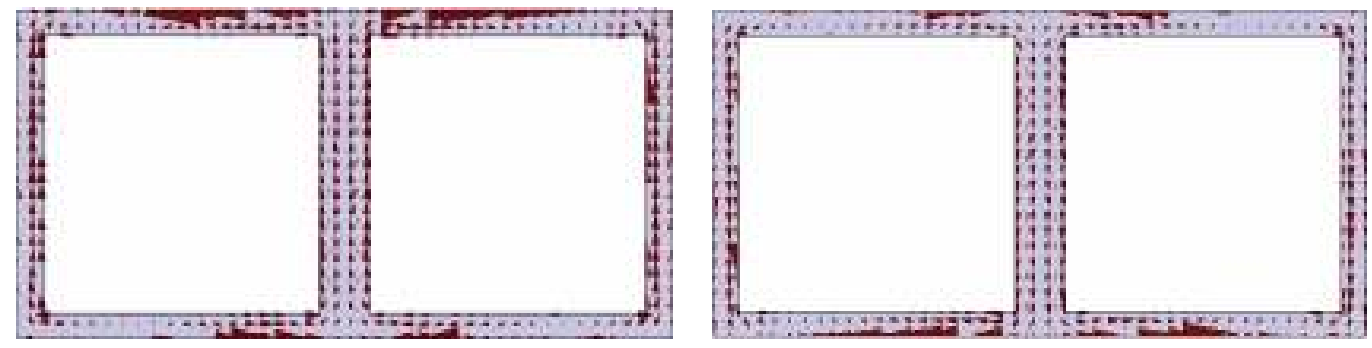

Figure 2: Current distribution of opposing metallic surfaces of the fishnet-based MTM. The left panel corresponds to the first metallic layer while the right one represents the second layer. The size of the arrows indicates the strength of the current.

In the experiment, the stacked structure is placed at the center of a WR-90 waveguide of which the input and output ports are connected to a network analyzer via two coaxial probes. After the full two-port calibration, the reflection and transmission data, both in amplitude and phase, are measured. Figure 3 shows the simulated and measured S-parameter data for the investigated structure. The step-function-like transmission spectrum with a high left-handed $(\mathrm{LH})$ peak in the step, in conjunction with a dip in the phase close to the same frequency, $10 \mathrm{GHz}$, are clear indications for double negative and fishnet behavior. 

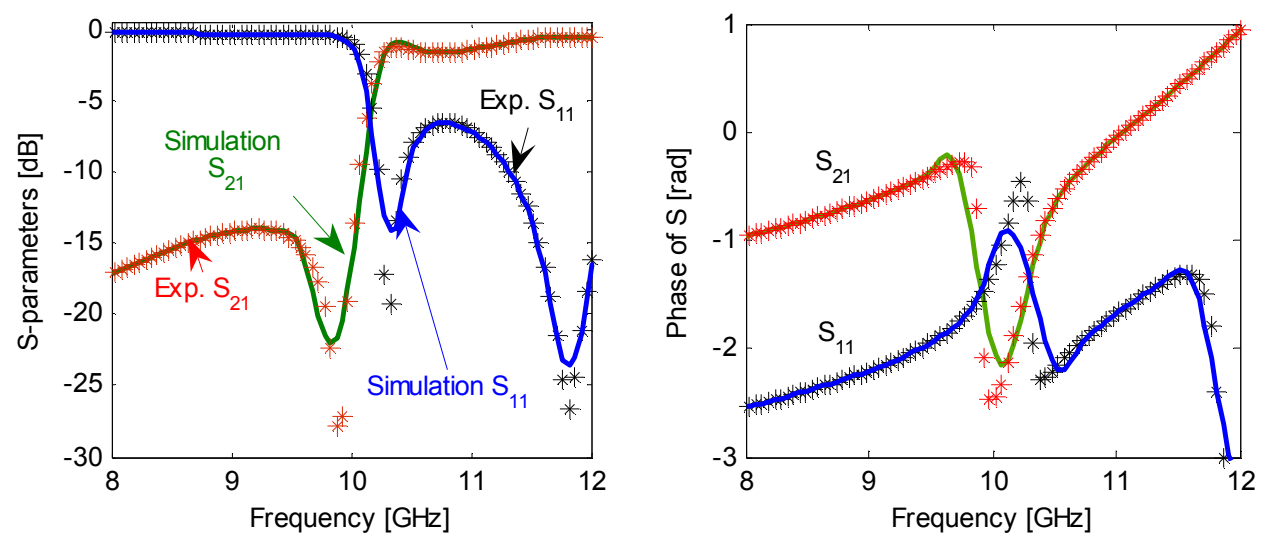

Figure 3: Simulated and measured S-parameters for the fishnet-based MTM.

The experimental and the simulation results are in good agreement with each other. The residual differences are likely to result from some deviations in the experiment from the idealized conditions of the simulations, a possible candidate being the dispersion of the dielectric constant of the substrate FR4 [18]. A second candidate is the fabrication tolerances related with the printed circuit board production, notably the etching process of the metal. Small changes of the lateral dimensions by a few microns during the manufacturing process can lead to a shift of the resonance on a megahertz scale. In addition, imperfect mounting of the multi-layer sample in the center of the waveguide are to be considered.

The effective material parameters of the investigated structure are obtained based on the assumption of an effectively homogeneous medium since the propagation wavelength is much larger than the periodicity of the structure in the propagation direction. The retrieval method is applied based on the method of images by properly selecting the boundary conditions which allow to treat the structure as an infinite homogeneous material. The equivalent structure is the combination of an infinite number of the repeated samples in all spatial directions. We expect that the structure will provide an anti-resonant behavior in the permittivity which is visible in our results. This behavior can also be seen in Refs. 5, 7, 8, and 19-26.

Figure 4 presents the extracted material parameters using the NRW method in the calculation [16, 17]. As it is seen from the figure, the structure provides simultaneously negative real parts of the permittivity and permeability in a band 
between $10.0 \mathrm{GHz}$ and $10.7 \mathrm{GHz}$ which covers the high transmission peak. However, the negative index band is larger and lies between $8.94 \mathrm{GHz}$ and $10.7 \mathrm{GHz}$ (see Figure 4, Zoom of $\operatorname{Re}(n))$. Note that only the real part of the permittivity is negative between $8.94 \mathrm{GHz}$ and $10 \mathrm{GHz}$. Although $\operatorname{Re}\{\varepsilon\}<0$ and $\operatorname{Re}\{\mu\}>0$, the refractive index can be negative when the imaginary term is large enough [4, 26-28]. This situation generally happens near the MTM resonance as can also be observed in the figure. Nevertheless, this type of negative refractive index does not support left-handed (LH) behavior. Thus, the negative refractive index has two regions which support either single negative (between $8.94 \mathrm{GHz}$ and $10 \mathrm{GHz}$ ) or double negative (between $10 \mathrm{GHz}$ and 10.7 GHz, LH region) characteristics. Also, the permittivity shows Drude-like behavior because of the coupling between the electric field and the copper strips as in the continuous-wires case. In addition, the permeability shows Lorentz-like behavior due to the strong resonant response to the magnetic field as in the magnetic resonators situation. Note that the anti-resonant behavior of the permittivity, the strong resonant response of the permeability, and the periodicity effects show that homogenous effective medium approximation can be applied with justification.

In order to verify the existence and validity of the negative index of refraction, a unique retrieval of effective parameters from the reflection and transmission data is obtained by enforcing the causality. The applied extraction method is based on the KK relations which have been used to calculate the index of refraction using calculated or measured data sets [29]. Note that, for practical reasons, KK integral has to be truncated, which yields some error. Nevertheless, the evaluation of the KK equation even for the finite bandwidth can provide approximate data for the verification of the actual data. As it is seen from Figure 4, there is a reasonable agreement between the results (simulation, experiment, and KK relation) for the real part of the effective refractive indexes which validates the existence of the negativeband and the anti-resonant behavior of the structure. 

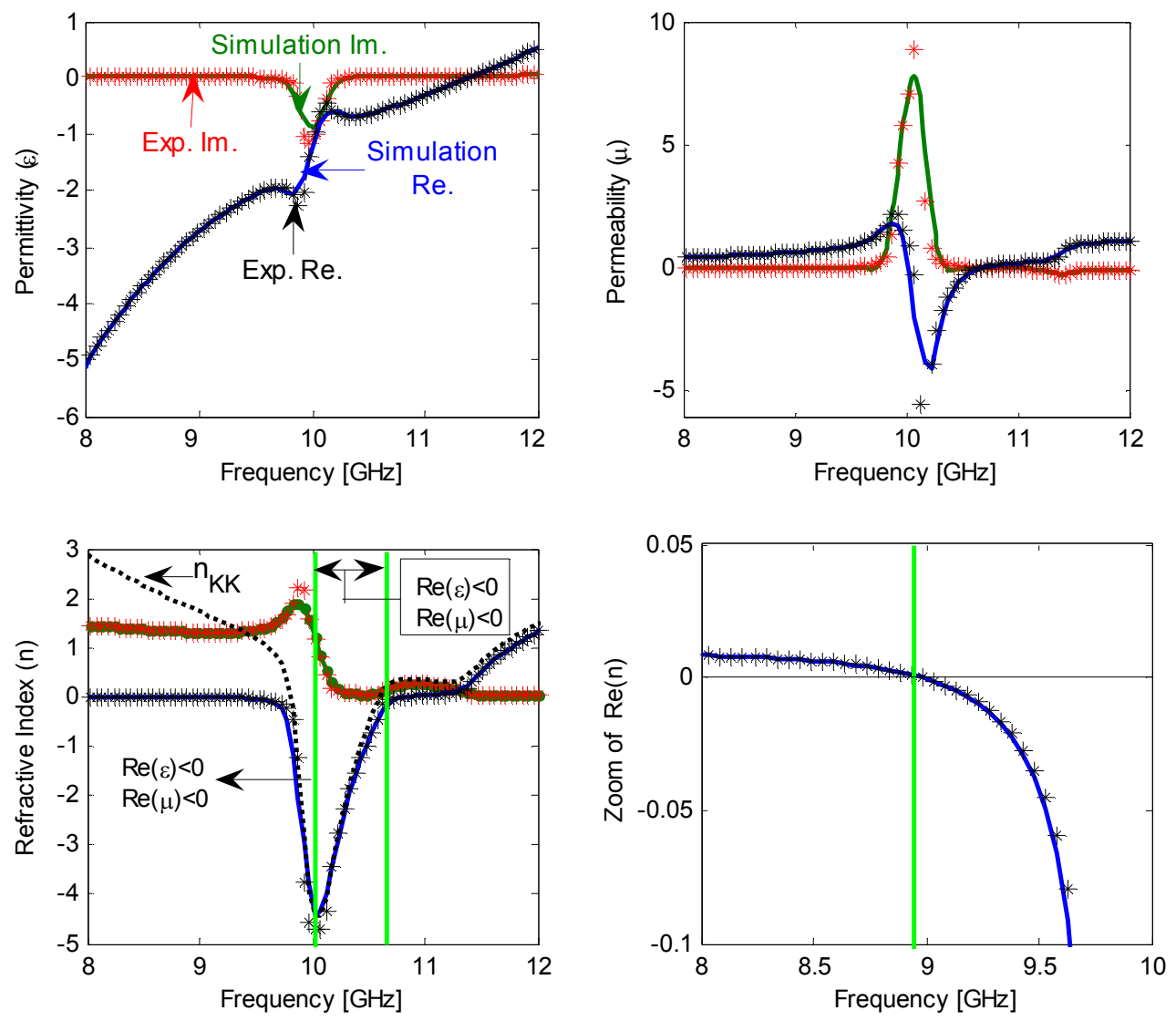

Figure 4: Effective material parameters as a function of frequency for the fishnetbased MTM.

Finally, Figure 5 shows the figure-of-merit (FOM, the ratio of $[-\operatorname{Re}\{\varepsilon\} / \operatorname{Im}\{\varepsilon\}])$ for the quality of the negative refraction of the investigated structure in terms of the loss. The proposed fishnet-based MTM of this study has a maximum FOM of 2.24 (simulated) and 2.13 (experimentally) at approximately $10.05 \mathrm{GHz}$ which is in the frequency band of the LH region. This indicates that the quality of the structure in terms of the loss and negative refraction is quite satisfactory for this kind of MTM. For comparison with the literature, we point the reader to Refs. [3-15]. For example, a FOM of approximately 6 is reported in Ref. 3, while it is approximately 3 in Ref. 5. In addition, one finds values of approximately 18 (simulated) and 3.5 (experimental) in Ref. 10, and of 4.2 in Ref. 12. As a result, one can say that the investigated structure behaves like a fishnet MTM with an acceptable FOM in the double negative region at microwave X-band frequencies. 


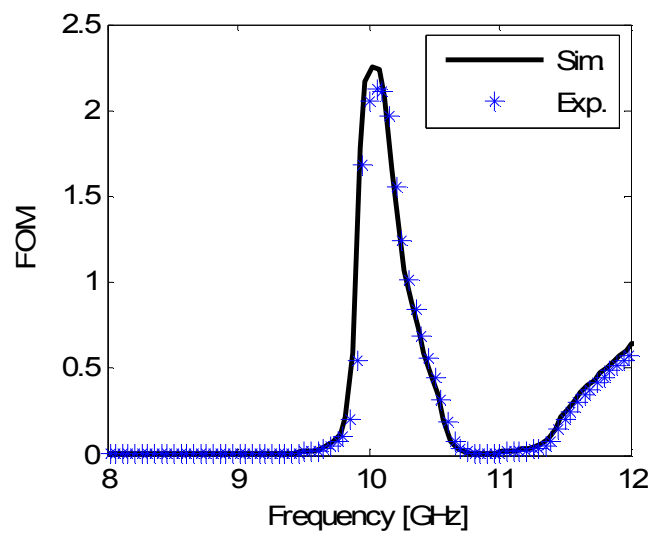

Figure 5: FOM as a function of frequency for the proposed MTM.

\section{Conclusion and Discussion}

In this study, a fishnet-based MTM has been introduced and the electromagnetic characteristics of the structure have been investigated, both numerically and experimentally, for the microwave X-band regime. The structure is designed to have no DC conductive connection between its metallic parts and the waveguide walls, and to exhibit double negative behavior at around $10 \mathrm{GHz}$. The currents, from one metal surface to the consecutive one, are anti-symmetric. Thus, they form a virtual current loop and generate a magnetic response yielding a negative permeability. The electric response which provides the negative permittivity is controlled by the structural parameters. The homogenous-effective-medium theory is applied to acquire the effective material parameters of the presented MTM. The single and double negative regions with evidence for a $\mathrm{LH}$ transmission peak are identified using the simulated and experimental results. The structure exhibits a relatively high FOM and opens opportunities notably with regard to waveguide miniaturization and for waveguidebased applications such as waveguide antennas and imaging systems in a waveguide. With regard to the latter, a waveguide with the proposed fishnet MTM can be designed for near-field imaging with the evanescent field from the interface, or exploiting the negative refractive index close to minus unity at around $10.5 \mathrm{GHz}--$ one can envision a lensing system in a multimode waveguide at microwave frequencies [30]. 


\section{Acknowledgement:}

C. S. acknowledges the COST Action MP0702 for the STSM grant; would like to thank to S. A. Tretyakov for being the host for the STSM, providing his facility for the experimental study, and his valuable comments; to C. R. Simovski for his helpful comments; and to O. Luukkonen, A. Karilainen, and P. Alitalo for their help during the experimental study.

\section{References}

[1] S. Tretyakov, Analytical Modeling in Applied Electromagnetics, Artech House Publishers, Norwood, MA, 2003.

[2] N. Engheta, and R. W. Ziolkowski, Metamaterials - Physics and Engineering Explorations, Wiley - IEEE Press, Piscataway, NJ, 2006.

[3] S. Zhang, W. Fan, K. J. Malloy, and S. R. J. Brueck, "Near-infrared double negative metamaterials," Optics Express, vol. 13, pp. 4922, 2005.

[4] S. Zhang, W. Fan, N. C. Panoiu, K. J. Malloy, R. M. Osgood, and S. R. J. Brueck, "Experimental demonstration of near-infrared negative-index metamaterials," Physical Review Letters, vol. 95, 137404, 2005.

[5] G. Dolling, Ch. Enkrich, M. Wegener, C. M. Soukoulis, and S. Linden, "Low-loss negative-index metamaterial at telecommunication wavelengths," Optics Letters, vol. 31, pp. 1800-1802, 2005.

[6] U. K. Chettiar, A. V. Kildishev, H.-K. Yuan, W. Cai, S. Xiao, V. P. Drachev, and V. M. Shalaev, "Dual-band negative index metamaterial: double negative at 813 nm and single negative at 772 nm," Optics Letters, vol. 32, pp. 1671-1673, 2007.

[7] M. Kafesaki, I. Tsiapa, N. Katsarakis, Th. Koschny, C. M. Soukoulis and E. N. Economou, "Left-handed metamaterials: The fishnet structure and its variations," Physical Review B, vol. 75, 235114, 2007.

[8] K. B. Alici and E. Ozbay, "Characterization and tilted response of a fishnet metamaterial operating at $100 \mathrm{GHz}$," Journal of Physics D: Applied Physics, vol. $41,135011,2008$.

[9] K. B. Alici and E. Ozbay, "A planar metamaterial: Polarization independent fishnet structure," Photonics and Nanostructures - Fundamentals and Applications, vol. 6, pp. 102-107, 2008. 
[10] J. Valentine, S. Zhang, T. Zentgraf, E. Ulin-Avila, D. A. Genov, G. Bartal, and X. Zhang, " Three-dimensional optical metamaterial with a negative refractive index," Nature, vol. 455, pp. 376-379, 2008.

[11] R. Marques, L. Jelinek, F. Mesa, and F. Medina, "Analytical theory of wave propagation through stacked fishnet metamaterials," Optics Express, vol. 17, pp. 11582-11593, 2009.

[12] C. Garcia-Meca, R. Ortuno, F. J. Rodriguez-Fortuno, J. Marti, and A. Martinez, "Double-negative polarization-independent fishnet metamaterial in the visible spectrum," Optics Letters, vol. 34, pp. 1603-1605, 2009.

[13] Z. Wei, Y. Cao, J. Han, C. Wu, Y. Fan, and H. Li, "Broadband negative refraction in stacked fishnet metamaterial," Applied Physics Letters, vol. 97, $141901,2010$.

[14] C. Menzel, T. Paul, C. Rockstuhl, T. Pertsch, S. Tretyakov, and F. Lederer, "Validity of effective material parameters for optical fishnet metamaterials," Physical Review B, vol. 81, 035320, 2010.

[15] L. Jelinek, R. Marques, and J. Machac, "Fishnet Metamaterials - Rules for refraction and limits of homogenization," Optics Express, vol. 18, pp. 1794017949, 2010.

[16] A. M. Nicolson, and G. Ross, "Measurement of the intrinsic properties of materials by time domain techniques," IEEE Transactions on Instrumentation and Measurement, vol. IM-19, pp.377-382, 1970.

[17] W. B. Weir, “Automatic measurement of complex dielectric constant and permeability at microwave frequencies," Proceedings of the IEEE, vo1.62, pp.3336, 1974.

[18] http://en.wikipedia.org/wiki/FR-4

[19] S. O'Brien and J. B. Pendry, "Photonic band-gap effects and magnetic activity in dielectric composites," Journal of Physics: Condensed Matter, vol. 14, pp. 40354044, 2002.

[20] S. O'Brien and J. B. Pendry, "Photonic band-gap effects and magnetic activity in dielectric composites,” Journal of Physics: Condensed Matter, vol. 14, pp. 40354044, 2002.

[21] D. R. Smith, S. Schultz, P. Markos, and C. M. Soukoulis, "Determination of effective permittivity and permeability of metamaterials from reflection and transmission coefficients," Physical Review B, vol. 65, 195104, 2002. 
[22] T. Koschny, P. Markos, D. R. Smith, and C. M. Soukoulis, "Resonant and antiresonant frequency dependence of the effective parameters of metamaterials," Physical Review E, vol. 68, 065602, 2003.

[23] P. Markos and C. Soukoulis, "Transmission properties and effective electromagnetic parameters of double negative metamaterials," Optics Express, vol. 11, pp. 649-661, 2003.

[24] S. O’Brien, D. McPeake, S.A. Ramakrishna, and J.B. Pendry, "Near infrared photonic bandgaps and nonlinear effects in negative magnetic metamaterials," Physical Review B, vol. 69, 241101, 2004.

[25] T. Koschny, P. Markos, D. R. Smith, and C. M. Soukoulis, "Reply to comments on "Resonant and antiresonant frequency dependence of the effective parameters of metamaterials"," Physical Review E, vol. 70, 048603, 2004.

[26] J. F. Zhou, L. Zhang, G. Tuttle, T. Koschny, and C. M. Soukoulis, "Negative index materials using simple short wire pairs," Physical Review B, vol. 73, 041101, 2006.

[27] M. W. McCall, A. Lakhtakia, and W.S. Weiglhofer, "The negative index of refraction demystified," European Journal of Physics, vol. 23, pp. 353-359, 2002.

[28] R.A. Depine and A. Lakhtakia, "A new condition to identify isotropic dielectricmagnetic materials displaying negative phase velocity," Microwave and Optical Technology Letters, vol. 41, pp. 315-316, 2004.

[29] V. V. Varadan and R. Ro, "Unique retrieval of complex permittivity and permeability of dispersive materials from reflection and transmitted fields by enforcing causality," IEEE Transactions on Microwave Theory and Techniques, vol. 55, pp. 2224-2230, 2007.

[30] A. Grbic, L. Jiang, and R. Merlin, "Near-Field Plates: Subdiffraction Focusing with Patterned Surfaces," Science, vol. 320, pp. 511-513, 2008. 